\title{
Editorial: Datasets for Brain-Computer Interface Applications
}

\author{
Ian Daly ${ }^{1 *}$, Ana Matran-Fernandez ${ }^{1}$, Davide Valeriani ${ }^{2}$, Mikhail Lebedev ${ }^{3,4}$ and \\ Andrea Kübler ${ }^{5}$ \\ ${ }^{1}$ Brain-Computer Interfacing and Neural Engineering Laboratory, School of Computer Science and Electronic Engineering, \\ University of Essex, Colchester, United Kingdom, ${ }^{2}$ Neurable Inc., Boston, MA, United States, ${ }^{3} \mathrm{~V}$. Zelman Center for \\ Neurobiology and Brain Restoration, Skolkovo Institute of Science and Technology, Moscow, Russia, ${ }^{4}$ Department of \\ Information and Internet Technologies of Digital Health Institute, I.M. Sechenov First Moscow State Medical University, \\ Moscow, Russia, ${ }^{5}$ Institute of Psychology, Julius Maximilian University of Würzburg, Würzburg, Germany
}

Keywords: brain computer interface, datasets, EEG, functional near infrared spectroscopy, electromyogram, ERP, steady-state visual evoked potential, affect (emotion)

\section{Editorial on the Research Topic}

\section{Datasets for Brain-Computer Interface Applications}

Non-invasive Brain-computer interfaces are an exciting new technology that provide a channel for communication between the brain and a computer system. They can be used as communication devices (Chaudhary et al., 2016; Brumberg et al., 2018), rehabilitation systems (Cervera et al., 2018), entertainment devices (Gürkök et al., 2017), and for a wide range of other applications (Finke et al., 2009; Makeig et al., 2011).

Research in non-invasive BCIs is developing rapidly and is a highly multidisciplinary field,

OPEN ACCESS

Edited and reviewed by: Michele Giugliano, International School for Advanced Studies (SISSA), Italy

*Correspondence: lan Daly i.daly@essex.ac.uk

Specialty section:

This article was submitted to Neural Technology,

a section of the journal

Frontiers in Neuroscience

Received: 28 June 2021

Accepted: 12 August 2021 Published: 29 September 2021

Citation:

Daly I, Matran-Fernandez A Valeriani $D$, Lebedev $M$ and Kübler $A$ (2021) Editorial: Datasets for

Brain-Computer Interface Applications.

Front. Neurosci. 15:732165 doi: 10.3389/fnins.2021.732165 involving, among others, neuroscientists, engineers, psychologists, computer scientists, and clinicians. Continuing development of BCI technology relies on advances made in each of these fields, which individually and collectively can contribute to improving all aspects of BCI systems including signal acquisition, processing, classification, and user interface design.

Many individual parts of a BCI system are typically first developed and evaluated on pre-existing datasets. However, there are only a few high quality publicly available datasets on which new systems, tools, and technologies can be evaluated and compared. For example, the publicly available BCI competition datasets (Sajda et al., 2003; Blankertz et al., 2004, 2006) provide an excellent set of resources for BCI researchers and have been widely used by numerous researchers to develop and evaluate new signal processing and classification methods (Arvaneh et al., 2013; Ghaemi et al., 2017; Lotte et al., 2018; Sakhavi et al., 2018; Zanini et al., 2018; Zhang et al., 2018). Yet, the relatively small size and number of such datasets introduce the risk of overfitting to methods developed and evaluated with these datasets. In other words, the reliability and reproducibility of BCI research is held back by a lack and sparsity of publicly available datasets.

This special issue provides a collection of descriptions of publicly available physiological datasets recorded during development, training, and evaluation of non-invasive BCI systems from BCI research labs around the world.

The collected datasets consist of signals recorded via a wide variety of modalities, including, but not limited to, electroencephalography (EEG), functional near infrared spectroscopy (fNIRS), electromyography (EMG), electrocardiography (ECG), galvanic skin response (GSR), skin temperature measures, respiration rates, and body movement data. Many datasets include multimodal recordings with combinations of two or more of these signal modalities.

Data from a wide variety of different BCI paradigms are described. These include development of novel event-related potential (ERP) and steady state visual evoked potential (SSVEP) based BCIs 
for communication, motor imagery BCIs, affective BCIs, collaborative BCIs, and neurofeedback-based BCIs for nicotine addiction, as well as resting-state data.

Data on ERP-based BCIs are provided by several authors. For example, Delijorge et al. describe an EEG-based P300-based robotic hand control BCI; Simões et al. provide a large EEG-based P300-based BCI dataset; Li et al. implemented an ERP-based BCI for communication.

Motor control-based BCIs and associated datasets are also included in this collection. For example, Brandl and Blankertz provide an EEG dataset recorded during motor imagery while distractions were presented to simulate day-to-day events experienced outside the lab. Schwarz et al. made an attempt to decode reach and grasp actions from the EEG. Ortega et al. collected a multimodal dataset comprising EEG, fNIRS, EMG, and movement data recorded during a force grip task.

A wide range of other types of EEG-based BCIs are also presented. These include a dataset for a BCI based on covert attention shifts (Reichert et al.) and an affective BCI based on neurofeedback (Charles et al.), as well as two BCIs based on the rapid serial visual presentation paradigm (Zhang et al.; Zheng et al.). The collection also includes a BCI for treating nicotine

\section{REFERENCES}

Arvaneh, M., Guan, C., Ang, K. K., and Quek, C. (2013). Optimizing spatial filters by minimizing within-class dissimilarities in electroencephalogrambased brain-computer interface. IEEE Transac. Neural Netw. Learn. Syst. 24, 610-619. doi: 10.1109/TNNLS.2013.2239310

Blankertz, B., Müller, K.-R., Curio, G., Vaughan, T. M., Schalk, G., Wolpaw, J. R., et al. (2004). The BCI Competition 2003: progress and perspectives in detection and discrimination of EEG single trials. IEEE Trans. Biomed. Eng. 51, 1044-1051. doi: 10.1109/TBME.2004.826692

Blankertz, B., Müller, K.-R., Krusienski, D. J., Schalk, G., Wolpaw, J. R., Schlögl, A., et al. (2006). The BCI competition. III: validating alternative approaches to actual BCI problems. IEEE Transac. Neural Syst. Rehabil. Eng. 14, 153-159. doi: 10.1109/TNSRE.2006.875642

Brumberg, J. S., Pitt, K. M., Mantie-Kozlowski, A., and Burnison, J. D. (2018). Brain-computer interfaces for augmentative and alternative communication: a tutorial. Am. J. Speech Lang. Pathol. 27, 1-12. doi: 10.1044/2017_AJSLP-16-0244

Cervera, M. A., Soekadar, S. R., Ushiba, J., Millán, J. D. R., Liu, M., Birbaumer, N., et al. (2018). Brain-computer interfaces for post-stroke motor rehabilitation: a meta-analysis. Ann. Clin. Transl. Neurol. 5, 651-663. doi: 10.1002/acn3.544

Chaudhary, U., Birbaumer, N., and Ramos-Murguialday, A. (2016). Braincomputer interfaces for communication and rehabilitation. Nat. Rev. Neurol. 12, 513-525. doi: 10.1038/nrneurol.2016.113

Finke, A., Lenhardt, A., and Ritter, H. (2009). The MindGame: a P300based brain-computer interface game. Neural Netw. 22, 1329-1333. doi: 10.1016/j.neunet.2009.07.003

Ghaemi, A., Rashedi, E., Pourrahimi, A. M., Kamandar, M., and Rahdari, F. (2017). Automatic channel selection in EEG signals for classification of left or right hand movement in Brain Computer Interfaces using improved binary gravitation search algorithm. Biomed. Signal Process. Control. 33, 109-118. doi: 10.1016/j.bspc.2016.11.018

Gürkök, H., Hakvoort, G., Poel, M., and Nijholt, A. (2017). Meeting the expectations from brain-computer interfaces. Comput. Entertain. 15:5. doi: 10.1145/2 633431

Lotte, F., Bougrain, L., Cichocki, A., Clerc, M., Congedo, M., Rakotomamonjy, A., et al. (2018). A review of classification algorithms for EEG-based brain-computer interfaces: a 10 year update. J. Neural Eng. 15:031005. doi: 10.1088/1741-2552/ $\mathrm{aab} 2 \mathrm{f} 2$ addiction via neurofeedback (Bu et al.) and a dataset of SSVEP signals (Liu et al.).

A diverse range of paradigms were used in this collection of studies. For example, von Lühmann et al. present a resting state fNIRS dataset, while Parent et al. provide a multimodal dataset, comprising EEG, ECG, and respiration activity, recorded during a range of physical activities and induced stress. Finally, Albuquerque et al. offer a multimodal dataset, comprising EEG, ECG, and GSR, recorded during a mental workload paradigm.

We expect that the collected datasets will enable novel developments and applications of BCI technology, as well as extensive validation studies of current and future BCIs.

\section{AUTHOR CONTRIBUTIONS}

All authors co-wrote the editorial and edited the Research Topic.

\section{FUNDING}

ML was supported by the Russian Science Foundation grant 21-75-30024.

Makeig, S., Leslie, G., Mullen, T., Sarma, D., Bigdely-Shamlo, N., and Kothe, C. (2011). "First demonstration of a musical emotion BCI," in International Conference on Affective Computing and Intelligent Interaction (Berlin; Heidelberg: Springer) 487-496.

Sajda, P., Gerson, A., Müller, K.-R. K.-R., Blankertz, B., and Parra, L. (2003). A data analysis competition to evaluate machine learning algorithms for use in braincomputer interfaces. IEEE Transac. Neural Syst. Rehabil. Eng. 11, 184-185. doi: 10.1109/TNSRE.2003.814453

Sakhavi, S., Guan, C., and Yan, S. (2018). Learning Temporal Information for Brain-Computer Interface Using Convolutional Neural Networks. IEEE Transac. Neural Netw. Learn. Syst. 29, 5619-5629. doi: 10.1109/TNNLS.2018.2789927

Zanini, P., Congedo, M., Jutten, C., Said, S., and Berthoumieu, Y. (2018). Transfer learning: a riemannian geometry framework with applications to brain-computer interfaces. IEEE Transac. Biomed. Eng. 65, 1107-1116. doi: 10.1109/TBME.2017.2742541

Zhang, Y., Wang, Y., Zhou, G., Jin, J., Wang, B., Wang, X., et al. (2018). Multikernel extreme learning machine for EEG classification in brain-computer interfaces. Expert Syst. Appl. 96, 302-310. doi: 10.1016/j.eswa.2017.12.015

Conflict of Interest: DV is employed by Neurable Inc.

The remaining authors declare that the research was conducted in the absence of any commercial or financial relationships that could be construed as a potential conflict of interest.

Publisher's Note: All claims expressed in this article are solely those of the authors and do not necessarily represent those of their affiliated organizations, or those of the publisher, the editors and the reviewers. Any product that may be evaluated in this article, or claim that may be made by its manufacturer, is not guaranteed or endorsed by the publisher.

Copyright (c) 2021 Daly, Matran-Fernandez, Valeriani, Lebedev and Kübler. This is an open-access article distributed under the terms of the Creative Commons Attribution License (CC BY). The use, distribution or reproduction in other forums is permitted, provided the original author(s) and the copyright owner(s) are credited and that the original publication in this journal is cited, in accordance with accepted academic practice. No use, distribution or reproduction is permitted which does not comply with these terms. 